\title{
Interpreting Results of Ethanol, Carbamazepine and Topiramate in Putrefied Postmortem Specimens: A Case Report
}

\author{
Maria Chiara David ${ }^{1}$, Lucia Broccoli ${ }^{2}$ and Luana Lionetto ${ }^{3}$ \\ 1. PhD Forensic Science -Department of Public Healthy Legal Medicine - University of Tor Vergata, 00133 Rome, Italy \\ 2. PhD Legal Medicine and Forensic Science, 00157 Rome, Italy \\ 3. PhD Advanced Molecular Diagnostics Unit (DiMA), IDI-IRCCS, Istituto Dermopatico dell'Immacolata, 00167 Rome, Italy
}

\begin{abstract}
CBZ (carbamazepine), CBZ-epox (carbamazepine-10,11-epoxide), TPR (topiramate) and ethanol were detected in 37-year-old woman buried in the wall founded eighteen months after death. The woman was in treatment with anticonvulsants. The cause of death was strangulation investigated by immunohistochemical with GPA (glycophorin A). Routine analyses for drugs of abuse, therapeutic drugs and volatiles were conducted. Carbamazepine, Topiramate and Alcohol were quantified in abdominal effusion, gastric wall, spleen, douglas fluid, skeletal muscles, endothoracic fluid, kidney, liver, heart and bone marrow. CBZ, CBZ-epox and TPR were recovered in samples deproteinized by acetonitrile spiked with DNSnVal as Internal Standard. Compounds were detected by HPLC-MS/MS. Alcohol was detected in any specimens by HS-GC/FID. CBZ concentrations were ranged from 0.49 in liver to $13.6 \mu \mathrm{g} / \mathrm{g}$ in endothoracic fluid; CBZ-Epox 0.46 in skeletal muscle, and $1.13 \mu \mathrm{g} / \mathrm{g}$ in Douglas fluid; TPR 0.11 in gastric wall and $1.23 \mu \mathrm{g} / \mathrm{g}$ in endothoracic fluid; alcohol from 0.17 in bone marrow to $0,75 \mathrm{mg} / \mathrm{g}$ in Douglas fluid. To our knowledge this is the first report of the presence of carbamazepine, topiramate, and alcohol in post mortem putrefied specimens.
\end{abstract}

Key words: Carbamazepine, topimarate, HPLC-MS/MS, postmortem, putrefied, saponified.

\section{Introduction}

It is often difficult to interpret analytical data obtained from post-mortem putrefied samples [1]. Several factors could affect the consistency of the results, which is particularly relevant when analyzing a decomposed body. Tissues are usually exposed for a long time and subject to different phenomena of biotransformation and decomposition which additionally depend on the environmental factors, and the time of exposure of the body [2, 3]. Thus, the interpretation of forensic data in putrefied tissues could be difficult due to drugs instability, degradation, post-mortem redistribution, drugs diffusion, external contamination, etc [4-6]. The choice of specimens that are useful for a toxicological analysis usually depends on the case, though the most commonly selected

Corresponding author: Drs. Maria Chiara David, Ph.D., forensic science mariachiara, research field: forensic science. specimens for drug analysis in routine cases are blood, urine, bile, vitreous humour, liver, and kidney [7]. However, in post-mortem forensic toxicology, different specimens can be used, as bone, muscle, brain, etc, which represents an important advantage. In the case of extreme putrefaction, specimens that can be collected mainly depend on the physical state of the body [8] and, under particular conditions such as saponification, biological fluids could be low-quality and altered and this would hinder the required analyses. In these cases, alternative specimens should be considered. Specimens are rarely ideal in saponification cases and, without a specialist knowledge, results should be cautiously interpreted, especially the significance of the results from samples in which drug levels are determined, as it.is difficult to find "therapeutic" levels of drugs in putrefied tissues.

Saponification is a special transformative 
phenomenon of conservative type, which only requires the water inside the body that occurs when the body is submerged in water or buried in moist soil. Similarly, it occurs when the body is placed inside a tightly closed container (e.g. in a metal box or a plastic bag), as the liquid inside the body ensures the required aqueous component [9]. Under these conditions internal organs are usually well preserved and it is often useful to achieve their macroscopic identification in the intact corpse. However, few cases related to drug research in saponified corpses have been published up to date. In this study, we described a case-report of a 37-year-old white woman found wrapped in plastic bags and blankets.

\section{A Case Report}

A 37-year- old woman was found at home buried in the wall, wrapped in plastic bags and blankets, eighteen months after her death. When a body is found, the forensic pathologist must perform a careful evaluation of all the available data, as the death could be related to suicide, homicide or could reveal the concealment of the corpse. In this case, at the autopsy, the body appeared in advanced state of putrefaction as shown in Fig. 1. Indeed, the body appeared saponified, the skin had a dry appearance, a yellow to brown colour and a leathery consistency. More specifically, the corpse was found at an incomplete state of saponification: transformative phenomena such as the emptiness of the orbital cavities after liquefaction of the eyeballs, and partial detachment of the attached follicles of the scalp were observed. Also internal organs of the chest and the abdomen were affected by advanced putrefaction process. Biological fluids such as blood, urine, bile, vitreous humour and gastric fluid were not found at the vessels and the cavity.

The potential cause of death was strangulation, which was investigated by immunohistochemical assay with GPA (glycophorin A) on the skin of the neck and histological analysis on the lungs. Routine analyses for drugs of abuse, therapeutics and volatile compounds were additionally performed. According to the clinical history, the patient was affected by "grand mal" seizures and she was under treatment with CBZ (carbamazepine) and TPR (topiramate). More specifically, the patient was under treatment with alternative daily doses of $100 \mathrm{mg}$ and $50 \mathrm{mg}$ TOPAMAX, and three daily doses of $200 \mathrm{mg}$ TEGRETOL. The disease was monthly monitored, though alcoholic beverages were usually consumed by the patient. Therefore, CBZ, CBZ-epox and TPR should be analysed in putrefied specimens in order to evaluate the contribution of antiepileptic drugs tissue concentrations on the epileptic status before her death.

Biological matrices (e.g. cerebrospinal fluid, bone, bone marrow, muscles) have been previously studied in putrefied corpses when available [10-13]. However, the scarce data regarding the analysis of these analytes on putrefied matrices together with the absence of fast and sensitive analytical methodologies warrant further research about these analytes on several matrices. In this case we have investigated the presence of prescribed drugs in order to elucidate the adherence of the patient to the corresponding therapy. With this

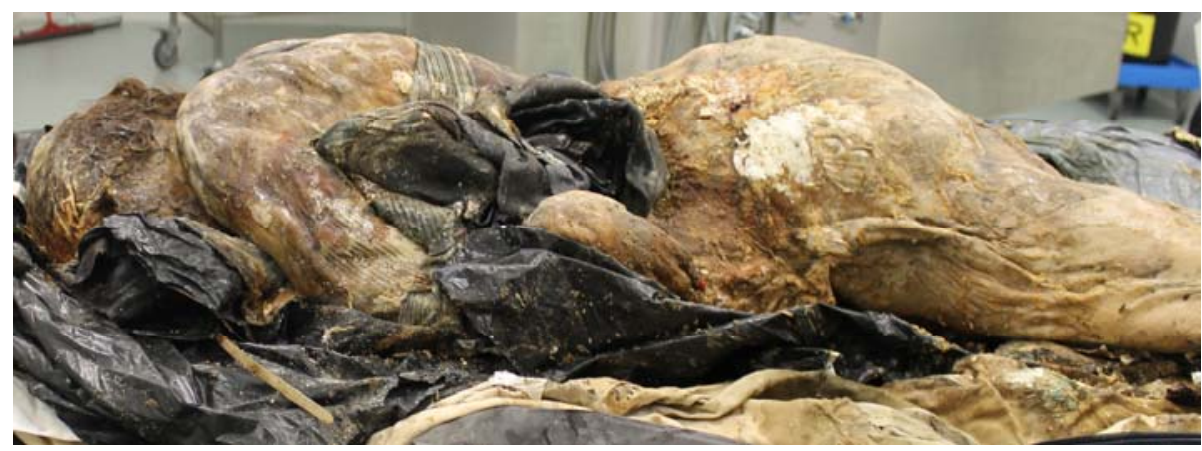

Fig. 1 The saponified body of the woman found buried in the wall. 
purpose, a previously validated methodology to detect CBZ, CBZ-epox in biological samples by HPLC-MS/MS (High Performance Liquid Chromatography-Mass Spectrometry) has been improved and validated to analyze these compounds, together with topiramate in several post-mortem specimens, such as abdominal effusion, gastric wall, spleen, Douglas fluid, skeletal muscle, endothoracic fluid, kidney, liver, heart and bone marrow.

\section{Materials and Methods}

\subsection{Chemical and Reagents}

Carbamazepine, carbamazepine-10,11-epoxide, topiramate and the IS (internal standard) dansyl-norvaline were purchased from Sigma Aldrich (St Louis, MO). HPLC-grade acetonitrile was purchased from Carlo Erba reagents (Milan, Italy) and formic acid was from Merck (Darmstadt, Germany). Water was deionized and filtered with a Milli-Q Plus apparatus (Millipore Corporation, Bedford, MA). Stock solutions $(10 \mathrm{mg} / \mathrm{mL})$ of CBZ, CBZ-epox and TPR were prepared by dissolving the pure analytes in the required solvent for the adequate solubilisation of each compound. Working solutions were prepared by diluting stock solutions with deionized water in order to obtain a final concentration of $1000 \mu \mathrm{g} / \mathrm{mL}$ for CBZ, CBZ-epox and TPR. Working solutions were additionally diluted to generate calibration curves ranging from 0.1 to $50 \mu \mathrm{g} / \mathrm{ml}$ for each compound. Working solution of DNSnVal $(100 \mu \mathrm{M})$ was prepared in acetonitrile for deproteinization. All solutions were stored at $-20{ }^{\circ} \mathrm{C}$ until final analysis by HPLC-MS/MS.

\subsection{Sample Preparation}

For chemical and toxicological analyses biological material collected through autopsy of the body with a scalpel were used. Samples from the cadaveric fluids were collected with a sterile syringe. All the received samples were stored at $-20^{\circ} \mathrm{C}$ until analysis.
Abdominal effusion, gastric wall, spleen, Douglas fluid, skeletal muscle, endothoracic fluid, kidney, liver, heart, and bone marrow samples were processed as following: briefly, the matrices were initially homogenized 1:3 with deionized water, and then the homogenized samples were then deproteinized twice. With this purpose, a total of $150 \mu \mathrm{L}$ acetonitrile solution containing DNSnVal as internal standard $(100 \mu \mathrm{M})$ was added to $50 \mu \mathrm{L}$ of the sample. The mixture was then vortexed up to $1 \mathrm{~min}$ and centrifuged at 13,500rpm for $10 \mathrm{~min}$. Fifty microliters of the supernatant were removed, added to $200 \mu \mathrm{L}$ of acetonitrile and, then, the sample was vortexed and centrifuged again. Fifty microliters of the second supernatant were mixed with $150 \mu \mathrm{L}$ of formic acid aqueous solution $(0.1 \%)$ and shaked for $10 \mathrm{sec}$. Finally, three consecutive injections $(10 \mu \mathrm{L})$ were run in the LC-MS/MS system.

\subsection{Methods}

CBZ, CBZ-epox and TPR were analysed by the LC-MS/MS procedure as a previous screening by applying a gas chromatography method was not sensitive enough to detect topiramate. Alternatively, a previously developed and validated LC-MS/MS methodology utilised to detect CBZ and CBZ-epox was further modified to detect TPR [14]. Compounds were separated on a reversed-phase High-Performance Liquid Chromatography System series 1100 (Agilent Technologies) and then quantified in the MRM (multiple reaction monitoring), in the positive mode by an API3200 tandem mass spectrometer (Applied Biosystems).

The optimal topiramate MRM transitions were selected for a sensitive and accurate MS/MS detection of the compound and, accordingly, the negative $\mathrm{m} / \mathrm{z}$ 338.3/77.9 has been selected for the quantification of this molecule. The Food and Drug Administration guidelines for bioanalytical method validation were used to fully validate this improved LC-MS/MS method, including selectivity, linearity, LOD (limit of 
detection), LLOQ (lower limit of quantification), recovery, within-day and between-day precision, and accuracy. Linear calibration curves $(0.10-50 \mu \mathrm{g} / \mathrm{mL}$ for carbamazepine; $\quad 0.05-20 \quad \mu \mathrm{g} / \mathrm{mL}$ for carbamazepine-10,11-epoxide; $0.10-100 \mu \mathrm{g} / \mathrm{mL}$ for topiramate) were obtained, with a mean correlation coefficient $>0.998$ for each compound. The accuracy and precision of each point on the standard curve were $>15 \% \mathrm{RE}$ and RSD and were used to calculate the accuracy and precision of the method by their analysis for 3 times in quintuplicate. The absolute recovery ranged from $95 \%$ to $102 \%$ for all analytes.

Routine analyses for drugs of abuse, therapeutic agents, and volatiles were additionally performed. In particular, enzyme immunoassay (TRIAGE, Biosite) and a basic drug extraction were used for drug screening and then confirmed by GC-MS. No other illicit or therapeutic drugs were detected in any of the analyzed specimens. Volatile compounds analysis was carried out by GC-FID/HS, and screening and quantification of ethanol, methanol, acetone and isopropanol were also determined. Alcohol was detected in each specimen by HS-GC/FID using isopropanol as internal standard.

\section{Results and Discussion}

The levels of carbamazepine, carbamazepine-10, 11-epoxide and topiramate found in each of the adulterated postmortem samples studied within the toxicological analysis at this particular case, are presented in Table 1.

Routine analyses for drugs of abuse, therapeutic drugs and volatiles were conducted in the presented case. The method described above was applied to the analysis of carbamazepine, carbamazepine-10, 11-epoxide and topiramate in several putrefied postmortem tissues. All the anticonvulsivant drugs and ethanol metabolites were successfully detected in biological material eighteen months after the patient's death.

In particular, we have investigated the concentration levels of the administered drugs in previously homogenated samples from abdominal effusion, gastric wall, spleen, Douglas fluid, skeletal muscle, endothoracic fluid, kidney, liver, heart and bone marrow. Carbamazepine is extensively bound to plasma proteins, and initial half-life values range from 25 to 65 hours, though these values decrease to $12-17$ hours when a subject is under chronic treatment (www.drugbank.ca). Carbamazepine is mainly metabolized in the liver, and carbamazepine-10, 11-epoxide represents its major hepatic metabolite. After oral administration, $72 \%$ and $28 \%$ of $\mathrm{CBZ}$ and CBZ-epox have been respectively found in urine and faeces (www.drugbank.ca). Daily therapeutic dose is $200 \mathrm{mg} /$ day and the therapeutic serum level is in the range of $4-12 \mu \mathrm{g} / \mathrm{ml}$ [15]. Moreover, higher hepatic levels of this drug have been detected in several cases of carbamazepine intoxication [15]. More specifically, concentrations ranging from 19.51 to $218.8 \mu \mathrm{g} / \mathrm{g}$ have

Table 1 Concentration of Carbamazepine, carbamazepine-10,11-epoxide and Topiramate detected in postmortem putrefied specimens.

\begin{tabular}{lllll}
\hline Specimen & CBZ $(\mu \mathrm{g} / \mathrm{g})$ & CBZ- epox $(\mu \mathrm{g} / \mathrm{g})$ & Topiramate $(\mu \mathrm{g} / \mathrm{g})$ & Alcohol $(\mathrm{mg} / \mathrm{g})$ \\
\hline Abdominal effusion & 0,93 & 1,00 & 0,92 & 0,35 \\
Gastric wall & 2,25 & 0,64 & 0,11 & 0,38 \\
Spleen & 4,48 & 0,91 & 0,85 & 0,34 \\
Douglas fluid & 2,62 & 1,13 & 0,83 & 0,75 \\
Skeletal muscle & 0,69 & 0,46 & 0,21 & 0,41 \\
Endothoracic fluid & 13,6 & 0,99 & 1,23 & 0,38 \\
Kidney & 2,64 & 1,10 & 0,68 & 0,28 \\
Liver & 0,49 & 0,67 & 0,64 & 0,34 \\
Heart & 4,37 & 0,70 & 0,95 & 0,22 \\
Bone marrow & 2,93 & 0,51 & 0,85 & 0,17 \\
\hline
\end{tabular}


been found in individuals who suffered from $\mathrm{CBZ}$ intoxication, whereas highly variable levels in a range of 1.57-362.5 $\mu \mathrm{g} / \mathrm{g}$ have been detected in the liver of individuals who suffered from different drug-drug or drug-xenobiotic interactions. Considering the low hepatic concentration of carbamazepine found in this case together with the absence of other xenobiotics or drugs, the intoxication by carbamazepine could be discarded. Additionally, the hepatic CBZ/CBZ-epox ratio was in accordance with a non-intoxicated state. Likewise, the levels of CBZ and CBZ-epox found in the kidney are lower than those detected in the acute intoxication cases [15].

Topiramate is well absorbed from the gastrointestinal tract, and peak plasma levels are usually obtained within 2-3 hours post-dose [16]. A small portion of drug is bound to plasma proteins. It is not extensively metabolized in the liver through hepatic hydroxylation, hydrolysis, and glucuronidation. The drug is primarily eliminated in the urine. Daily therapeutic dosage is 400-1000 $\mathrm{mg}$ /day [16] and the therapeutic plasma level is $2-25$ $\mu \mathrm{g} / \mathrm{ml}$ [17]. Few case reports on topiramate levels in post-mortem specimens have been published until now. We have found much lower topiramate concentrations in both liver and kidney when compared with previous cases of intoxication described in literature [16, 18, 19]. In particular, liver concentrations of $29 \mu \mathrm{g} / \mathrm{g}$ [16] and $140 \mu \mathrm{g} / \mathrm{g}$ [19] were found in previous studies, which are higher than the value detected in this study $(0,64 \mu \mathrm{g} / \mathrm{g})$, as well as the kidney concentrations $(55 \mu \mathrm{g} / \mathrm{g}$ vs $0,68 \mu \mathrm{g} / \mathrm{g})$ [18]. Nevertheless, these levels were detected in previous cases of topiramate intoxication as no data on post-mortem therapeutic levels for comparisons are available. However, the levels of Carbamazepine, carbamazepine-10, 11-epoxide and Topiramate found in the postmortem tissues analyzed in this study suggest long-term administration of the anticonvulsivant drugs, which is in accordance with the medical history.
The presence of ethanol was performed by a gas chromatography coupled to ionization detector with headspace sampling (HS-GC). The specimens were 1:2 diluted with an aqueous solution of the internal standard n-propanol in order to reduce the matrix effect. In this case, the body was saponified and the possibility of ethanol production or degradation was a dilemma. It is important to know water and lipid content in unusual tissues and the stability of ethanol after sampling in order to interpret the results, mainly if the corpse has undergone decomposition. Results have a typical post-mortem redistribution pattern in all the specimens analysed. To reach a correct diagnosis of whether a person was under the influence of alcohol it is needed to compare the concentrations of ethanol in several body fluid, but in this case no fluid such as urine or vitreous help us to evaluate the origin of ethanol to support the evidence of antemortem alcohol ingestion. Even if she used to drink alcoholic beverages, the condition of the body, the time between death and autopsy, the environmental conditions and the nature of the specimen collected for analysis made possible that the ethanol detected had been produced after death by microbial activity and fermentation of glucose [20].

In particular, the important issue is to understand if the concentrations of alcohol and drugs in biological samples properly reflect the concentrations at the time of death. Between death and specimen collection, drug concentrations in tissues, organs or body fluids may vary greatly. Changes in drug concentrations in post-mortem specimens may be caused by a number of factors (e.g. hydrolysis, bacterial activity and redistribution). The application of analytical techniques in post-mortem toxicology is often more difficult than in other forms of forensic toxicology due to the variable and often degraded nature of the specimens and the diverse range of specimens available for analysis.

At this special case, some interesting results were found when the analyses were carried out. Especially 
as it was the first time, up to our knowledge, that anticonvulsivants were analyzed and successfully found in such particular samples and conditions. Additionally, the drugs concentrations determined in this work would add valuable information on the forensic side of this study as it could potentially corroborate if patient took the prescribed drugs.

The methods applied on putrefied specimens gave results according to the dynamics of the events that led to the woman's death, as it was found at the end of the first instance, which culminated in the murder conviction of the accused. Given the results of the investigation, the autopsy and the toxicological findings, the medical examiner ruled the death to be a result of strangulation. In fact, the analytes concentrations appeared to be within the therapeutic range in each specimens, and the toxicological results confirmed the discordance between the morphological findings and the potential occurrence of an acute intoxication. Nevertheless, the results found in this study could not be compared with reference carbamazepine and topiramate concentrations in previously reported studies. Moreover, it should also be considered that the samples collected from eighteen months putrefied corpse could not be perfectly homogeneous and, consequently, the results should be cautiously interpreted.

\section{Conclusion}

Post-mortem toxicology is a stimulating field for the potential number of substances to be present and for the possible decomposition state of specimens other than the difficulties in interpreting the analytical results. These problems require interesting analytical answer. In forensic and toxicological literature there are few case reports about "therapeutic" concentrations of molecules in adulterated post-mortem specimens.

In postmortem testing it is important to compare drug concentrations in several samples, even if reference values of concentration in tissues are missing.

The measurements of drug concentration in postmortem putrefied unconventional specimens have a limited meaning except in the context of medical history, necropsy findings and circumstances surrounding death. We hope that this study will contribute to improve the field of forensic toxicology. To our knowledge this is the first report of the presence of carbamazepine, carbamazepine-10, 11-epoxide and topiramate in post-mortem saponified alternative specimens.

\section{References}

[1] Morini, L., Vignali, C., Tricomi, P., and Groppi, A. 2015. "Analytical Challenge in Postmortem Toxicology Applied to a Human Body Found into a Lake after Three Years Immersion.” J. Forensic Sci. 60 (5): 1383-6.

[2] Rosier, E., Loix, S., Develter, W., Voorde, W. V. D., Tytgat, J., et al. 2015. "The Search for a Volatile Human Specific Marker in the Decomposition Process." PLOS One 10 (9): $\mathrm{e} 0137341$.

[3] Skopp, G. 2010. "Postmortem Toxicology." Forensic Sci. Med. Pathol. 6 (4): 314-25.

[4] Drummer, O. H. 2007. "Post-mortem Toxicology." Forensic Sci. Int. 165 (2-3): 199-203.

[5] Han, E., Kim, E., Hong, H., Jeong, S., Kim, J., et al. 2012 "Evaluation of Postmortem Redistribution Phenomena for Commonly Encountered Drugs." Forensic Sci. Int. 219 (1-3): 265-71.

[6] Kennedy, M. C. 2010. "Post-mortem Drug Concentrations." Intern. Med. J. 40 (3): 183-7.

[7] Drummer, O. H. 2007. "Requirements for Bioanalytical Procedures in Postmortem Toxicology." Anal. Bioanal. Chem. 388 (7): 1495-503.

[8] Dinis-Oliveira, R. J., Carvalho FDuarte, J. A., Remiao, F., Marques, A., Santos, A., et al. 2010. "Collection of Biological Samples in Forensic Toxicology." Toxicol. Mech. Methods 20 (7): 363-414.

[9] Forbes, S. L., Wilson, M. E. A., and Stuart, B. H. 2011. "Examination of Adipocere Formation in a Cold Water Environment.” Int. J. Legal. Med. 125 (5): 643-50.

[10] Keller, T., Schneider, A., and Tutsch-Bauer, E. 1999. "GC/MS Determination of Zolpidem in Postmortem Specimens in a Voluntary Intoxication.” Forensic Sci. Int. 106 (2): 103-8.

[11] Bynum, N. D., Poklis JL Gaffney Kraft, M., Garside, D., and Ropero Miller, J. D. 2005. "Postmortem Distribution of Tramadol, Amitriptyline, and Their Metabolites in a Suicidal Overdose.” J. Anal. Toxicol. 29 (5): 401-6. 
[12] Jaffe, P. D., Batziris, H. P., Hoeven, P. V. D., and Desilva, D. 1999. "M Mcintyre. A Study Involving Venlafaxine Overdoses: Comparison of Fatal and Therapeutic Concentrations in Postmortem Specimens.” J. Forensic. Sci. 44 (1): 193-6.

[13] Vance, C., and McIntyre, I. M. 2009. "Postmortem Tissue Concentrations of Olanzapine.” J. Anal. Toxicol. 33 (1): $15-26$.

[14] Lionetto, L., Casolla, B., and Cavallari, M., et al. 2012. "High-Performance Liquid Chromatography-Tandem Mass Spectrometry Method for Simultaneous Quantification of Carbamazepine, Oxcarbazepine, and Their Main Metabolites in Human Serum." Ther Drug Monit. 34 (1): 53-8.

[15] Klys, M., and Bystrowska, B. G. B. 2003. "Postmortem Toxicology of Carbamazepine.” J. Anal. Toxicol. 27 (4): 243-8.
[16] Mozayani A., and Carter, J. R. 1999. "Distribution of Topiramate in a Medical Examiner's Case." J. Anal. Toxicol. 23: 556-8.

[17] Levine, B., Rebecca Jufer, P., Clare, N., Kisha, F., and David, F. 2010. "Tissue Distribution of Newer Anticonvulsant Drugs in Postmortem Cases." J. Anal. Toxicol. 34 (8): 506-9.

[18] Beer, B., Libiseller, K., Oberacher, H., and Pavlic, M. 2010. "A Fatal Intoxication Case Involving Topiramate." Forensic Sci. Int. 202 (1-3): e9-11.

[19] Langman, L. J., and Kaliciak HABoone, S. A. 2003. "Fatal Acute Topiramate Toxicity." J. Anal. Toxicol. 27 (5): 323-4.

[20] Kugelberg, F. C., and Jones, A. W. 2007. "Interpreting Results of Ethanol Analysis in Postmortem Specimens: A Review of the Literature." Forensic Sci. Int. 165 (1): $10-29$. 